\title{
Histone demethylase JARID1B promotes cell proliferation but is downregulated by $\mathrm{N}$-Myc oncoprotein
}

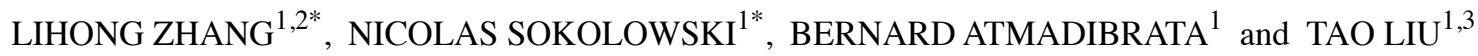 \\ ${ }^{1}$ Children's Cancer Institute Australia for Medical Research, Sydney, NSW 2031, Australia; \\ ${ }^{2}$ Department of Anatomy, Histology and Embryology, School of Basic Medical Sciences, Fudan University, \\ Shanghai 200032, P.R. China; ${ }^{3}$ School of Women's and Children's Health, UNSW Medicine, \\ University of New South Wales, Sydney, NSW 2031, Australia
}

Received November 30, 2013; Accepted December 27, 2013

DOI: $10.3892 /$ or.2014.3006

\begin{abstract}
Myc oncoproteins induce tumor initiation and promote tumor progression by modulating gene transcription. We have previously shown that N-Myc represses gene transcription by recruiting histone deacetylases to Sp1-binding site-enriched regions of target gene promoters. The histone demethylase JARID1B plays a dual role in cancer. In the present study, we examined published microarray gene expression datasets and found that JARID1B was commonly repressed by Myc oncoproteins and histone deacetylases in cancer cell lines of various organ origins. Chromatin immunoprecipitation assays demonstrated that N-Myc repressed JARID1B expression by direct binding to the Sp1-binding site-enriched region of the JARID1B gene promoter, and cell proliferation assays showed that transcriptional repression of JARID1B reduced neuroblastoma cell proliferation. Our findings suggest that Myc-mediated transcriptional repression of JARID1B counterintuitively inhibits Myc-regulated cell proliferation and potentially tumorigenesis.
\end{abstract}

\section{Introduction}

Neuroblastoma, which originates from precursor neuroblast cells of the sympathetic nervous system, is the most common solid tumor in children under the age of five. $M Y C N$ oncogene amplification and consequent N-Myc mRNA and protein overexpression occur in a quarter to a third of tumor tissues and correlate with poor prognosis in neuroblastoma patients $(1,2)$.

The structurally related Myc oncoproteins, N-Myc and c-Myc, exert oncogenic effects by modulating gene transcrip-

Correspondence to: Dr Tao Liu, Children's Cancer Institute Australia for Medical Research, Randwick, Sydney, NSW 2031, Australia

E-mail: tliu@ccia.unsw.edu.au

${ }^{*}$ Contributed equally

Key words: histone demethylase, JARID1B, N-Myc, neuroblastoma, gene expression tion. Myc oncoproteins activate gene transcription by direct binding to Myc-responsive element E-boxes at target gene promoters $(3,4)$, and repress gene transcription by forming transcriptional repressor complexes with histone deacetylases (HDACs), including the class I HDAC1 and HDAC2, the class II HDAC5 as well as the class III SIRT1 and SIRT2, at Sp1-binding sites of target gene promoters (5-9). Moreover, epigenetic alterations, such as histone H3 lysine 4 (H3K4) trimethylation at target gene promoter regions, are considered essential for Myc-mediated transcriptional activation (10).

JARID1B, also known as KDM5B and PLU1, is a Jumonji $\mathrm{C}$ domain-containing histone demethylase that specifically demethylates tri- and di-methylated histone $\mathrm{H} 3 \mathrm{~K} 4$ $(11,12)$. JARID1B promotes cell differentiation during development, as JARID1B is essential for neuronal differentiation of embryonic stem cells (13) and knocking down JARID1B is associated with reduced levels of differentiation-associated genes in hematopoietic stem cells (14). However, JARID1B plays a dual role in cancer.

In the present, study we identified JARID1B as one of the genes significantly repressed by Myc, and examined whether repression of JARID1B contributed to Myc-mediated cancer phenotypes.

\section{Materials and methods}

Cell culture. Neuroblastoma BE(2)-C cells were cultured in Dulbecco's modified Eagle's medium supplemented with $1 \%$ L-glutamine and $10 \%$ fetal calf serum. Kelly cells were grown in RPMI-1640 supplemented with 1\% L-glutamine and 10\% fetal calf serum.

siRNA transfection. Cells were transfected with AllStars negative control small interfering RNAs (siRNAs), N-Myc siRNAs or JARID1B siRNAs (Qiagen, Hamburg, Germany) using Lipofectamine ${ }^{\circledR} 2000$ reagent (Life Technologies, Grand Island, NY, USA) as we previously described (5-7,9).

Real-time RT-PCR. Following siRNA transfections, RNA was extracted from cells using PureLink RNA Mini kit (Life Technologies) according to the manufacturer's instructions. Synthesis of cDNA from RNA samples was carried out using 
Table I. JARIDIB gene expression is reactivated after reduction in Myc or histone deacetylase expression in a range of cancer cell lines.

\begin{tabular}{lllll}
\hline Cell lines & $\begin{array}{c}\text { Treatment or siRNA } \\
\text { transfection }\end{array}$ & $\begin{array}{c}\text { Microarray } \\
\text { platform }\end{array}$ & $\begin{array}{c}\text { JARID1B } \\
\text { gene expression }\end{array}$ & \multicolumn{1}{c}{ Refs. } \\
\hline BE(2)-C neuroblastoma cells & N-Myc siRNA & Affymetrix & $\uparrow$ & Marshall et al (6) \\
KMS11, MM.1S, OPM1 & JQ1 treatment & Affymetrix & $\uparrow$ Delmore et (17) \\
multiple myeloma cells & JQ1 treatment & Affymetrix & $\uparrow$ & Banerjee et al (18) \\
J-Lat T cells & HDAC2 siRNA & Affymetrix & $\uparrow$ & Marshall et al (6) \\
BE(2)-C neuroblastoma cells & SIRT2 siRNA & Affymetrix & $\uparrow$ & Liu et al $(9)$ \\
BE(2)-C neuroblastoma cells & SIRT1 siRNA & Affymetrix & $\uparrow$ & Marshall et al (7) \\
BE(2)-C neuroblastoma cells &
\end{tabular}

M-MLV reverse transcriptase (Invitrogen, Carlsbad, CA, USA). Real-time RT-PCR was performed using SYBR-Green PCR Master Mix (Life Technologies) and Applied Biosystems 7900 as we previously described (5-7,9). The expression of actin mRNA was employed as loading controls.

Immunoblot analysis. For the analysis of protein expression, cells were lysed, protein extracted and separated by gel electrophoresis. After western transfer, membranes were probed with a mouse anti-N-Myc antibody (1:1,000; Santa Cruz Biotechnology, Santa Cruz, CA, USA) or a rabbit anti-JARID1B antibody (1:100, Santa Cruz Biotechnology), followed by a horseradish peroxidase-conjugated anti-mouse (1:10,000, Santa Cruz Biotechnology) or anti-rabbit (1:2,000, Santa Cruz Biotechnology) antibody. Protein bands were visualized with SuperSignal (Pierce, Rockford, IL, USA). The membranes were lastly re-probed with an anti-actin antibody (Sigma, St. Louis, MO, USA) as loading controls.

Chromatin immunoprecipitation (ChIP) assays. ChIP assays were performed with an anti-N-Myc antibody (Merck Millipore, Billerica, MA, USA) or a control antibody and PCR with primers targeting negative control region (-2108 bp to -1946 bp upstream of the transcription start site) or Sp1-binding site-enriched region (-109 bp to -9 bp upstream of the transcription start site) of the JARID1B gene. Fold enrichment of the JARID1B gene core promoter region by the anti-N-Myc antibody was calculated by dividing the PCR product from the Spl-binding site-enriched region by the PCR product from the negative control region. The sequences of primers targeting the negative control region were 5'-GCAGCTGAGAATTGGG AAAG-3' (forward primer) and 5'-TCGGGAGAGCGTTGA CTATT-3' (reverse primer), and the sequences of primers targeting the JARID1B core promoter region were 5'-GTGGGG TGGGACTCTTTTTC-3' (forward primer) and 5'-CAGCAC CTTGGGCTTTTTC-3' (reverse primer).

Alamar blue assays. Alamar blue assays were carried out as we previously described (15). Briefly, cells were transfected with various siRNAs in 96-well plates. After $72 \mathrm{~h}$, cells were incubated with Alamar Blue (Invitrogen) for $6 \mathrm{~h}$, and the plates were read on a microplate reader at 570/595 nm. Results were calculated according to optical density absorbance units and expressed as percentage changes in viable cell numbers.

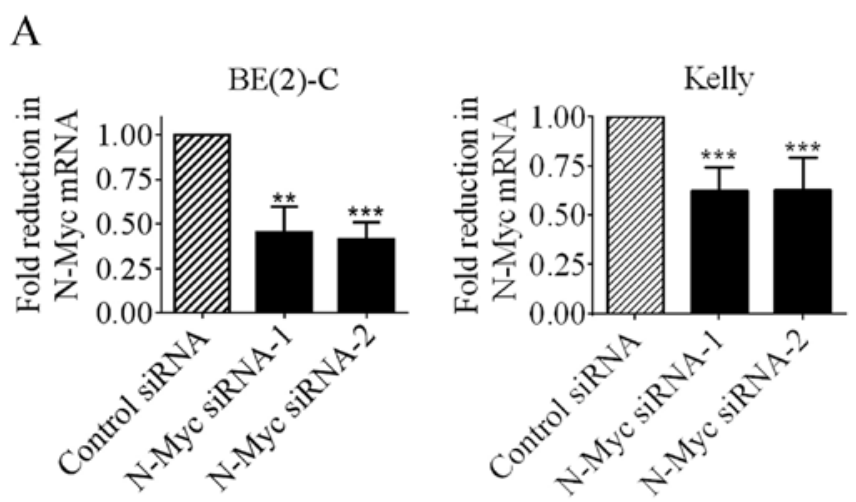

B

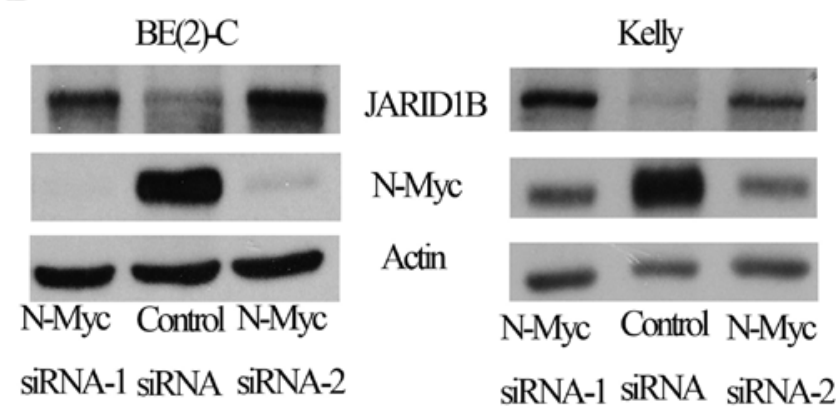

$\mathrm{C}$
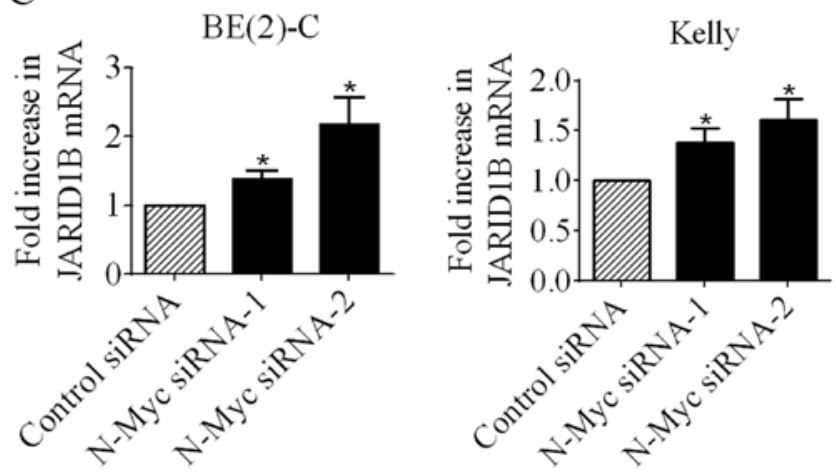

Figure 1. N-Myc suppresses JARID1B expression in neuroblastoma cells. $\mathrm{BE}(2)-\mathrm{C}$ and Kelly cells were transfected with AllStars negative control siRNA, N-Myc siRNA-1 or N-Myc siRNA-2 for $48 \mathrm{~h}$, followed by RNA and protein extraction, real-time RT-PCR and immunoblot analyses of (A) N-Myc mRNA, (B) N-Myc protein, (C) JARID1B mRNA and (B) JARID1B protein expression. Error bars represent standard error. ${ }^{*} \mathrm{P}<0.05,{ }^{* *} \mathrm{P}<0.01$ and ${ }^{* * * *} \mathrm{P}<0.001$ 


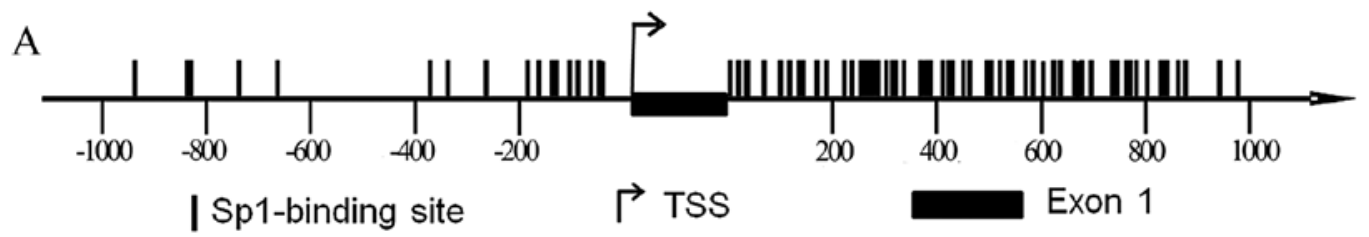

B

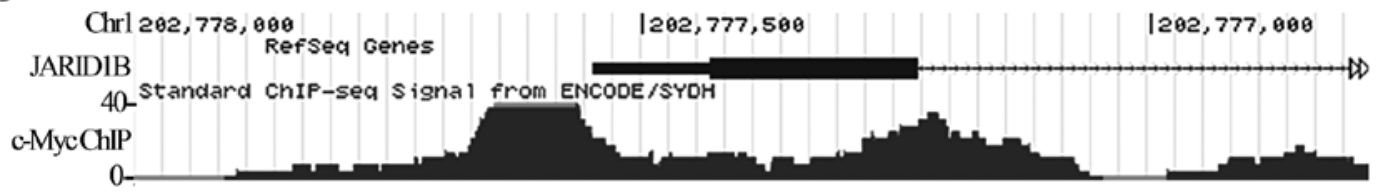

C

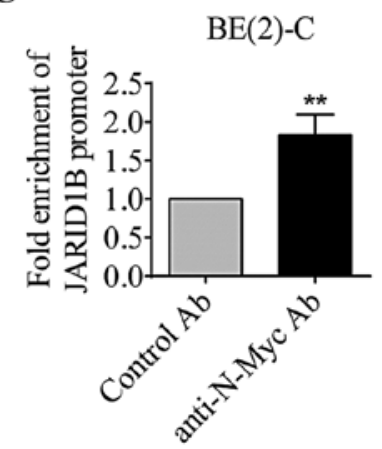

Figure 2. N-Myc suppresses JARID1B expression by direct binding to the JARID1B gene promoter. (A) Schematic representation of the JARID1B gene promoter. TSS represents transcription start site, and I represents Sp1-binding site. (B) ChIP-sequencing data from K562 cells generated by Dr Michael Snyder's group at Yale University for the ENCODE/SYDH project. Chr1 represents chromosome 1. (C) ChIP assays were performed with a control or an anti-N-Myc antibody (Ab) and primers targeting a negative control region or the JARID1B gene core promoter region enriched of Sp1-binding sites in BE(2)-C cells. Fold enrichment of the JARID1B gene core promoter by the control or the anti-N-Myc antibody was calculated by dividing PCR products from primers targeting the JARID1B gene core promoter by PCR products from primers targeting the negative control region. Fold enrichment by control antibody was artificially set as 1.0. Error bars represent standard error. ${ }^{* *} \mathrm{P}<0.01$.
Statistical analysis. The experiments were conducted at least 3 times. Data are presented as means \pm standard error. Differences were analyzed for significance using ANOVA among groups or unpaired t-test for two groups. A probability value of 0.05 or less was considered to indicate a statistically significant difference.

\section{Results}

JARIDIB gene expression is reactivated after reduction in Myc or histone deacetylase expression in a range of cancer cell lines. The interaction between c-Myc protein and JARID1B protein has been proposed to play an important role in c-Myc-mediated cell growth (16). We, therefore, examined the factors which modulated JARID1B gene expression in published microarray gene expression datasets. As shown in Table I, knocking down N-Myc expression with siRNAs significantly upregulated JARID1B mRNA expression in MYCN oncogene-amplified BE(2)-C neuroblastoma cells $(6,8)$. Consistently, JARID1B was one of the genes most markedly upregulated in c-Myc overexpressing KMS11, MM.1S and OPM1 multiple myeloma cells as well as J-Lat T cells after treatment with the BET bromodomain inhibitor JQ1, which exerted anticancer effects by repressing c-Myc gene transcription $(17,18)$. While histone deacetylases are well known to form transcriptional repressor complexes with Myc oncoproteins at Myc target gene promoters, knocking down the expression of the class I histone deacetylase HDAC2 (6), the class III histone deacetylase SIRT1 (7) or SIRT2 (9) with siRNAs upregulated JARID1B gene expression in neuroblastoma $\mathrm{BE}(2)-\mathrm{C}$ cells (Table I). The published microarray gene expression data suggest that JARID1B is a transcriptional target gene of Myc oncoproteins and histone deacetylases in a range of cancer cell lines.
$N$-Myc suppresses JARIDIB expression in neuroblastoma cells. To validate the microarray data that N-Myc suppressed JARID1B gene expression, we transfected the MYCN oncogene-amplified BE(2)-C and Kelly human neuroblastoma cells with control siRNA or two independent siRNAs targeting different regions of N-Myc mRNA, N-Myc siRNA-1 or N-Myc siRNA-2, followed by real-time RT-PCR and immunoblot studies of N-Myc and JARID1B. As shown in Fig. 1A and B, both N-Myc siRNAs efficiently knocked down N-Myc mRNA and protein expression in the two neuroblastoma cell lines. Of note, N-Myc siRNA-1 and N-Myc siRNA-2 increased the expression of JARID1B mRNA (Fig. 1C) and protein (Fig. 1B). The results confirm that N-Myc represses JARID1B mRNA and protein expression.

$N$-Myc suppresses JARIDIB expression by direct binding to the JARIDIB gene promoter. We previously showed that $\mathrm{N}-\mathrm{Myc}$ represses gene transcription by binding to Spl-binding site-enriched regions of target gene promoters $(5-7,9)$. To understand whether N-Myc could directly repress JARID1B gene transcription, we firstly analyzed transcription factor binding sites at the JARID1B gene promoter with Gene-Regulation software (http://www.gene-regulation.com/pub/programs/ alibaba2/index.html). Results showed that Sp1-binding sites were enriched at $-179 \mathrm{bp}$ to $-56 \mathrm{bp}$ upstream of the JARID1B gene transcription start site as well as +4 bp to +873 bp of the JARID1B intron 1 (Fig. 2A). We then examined a c-Myc chromatin immunoprecipitation-sequencing (ChIP-Seq) dataset, which was generated by Dr Michael Snyder's group at Yale University for the ENCODE/SYDH project (The Encyclopedia of DNA Elements/Stanford/Yale/USC/Harvard genome project) and deposited at the University of California Santa Cruz Genome Browser website. As shown in Fig. 2B, the ChIP-seq data showed that c-Myc oncoprotein bound to the 
A

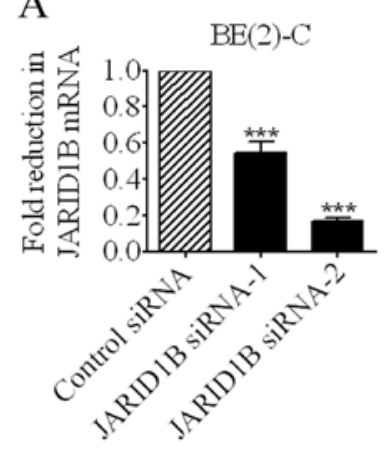

B

$\mathrm{BE}(2)-\mathrm{C}$

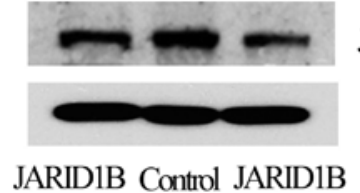

SIRNA-1 SiRNA SIRNA-2

C

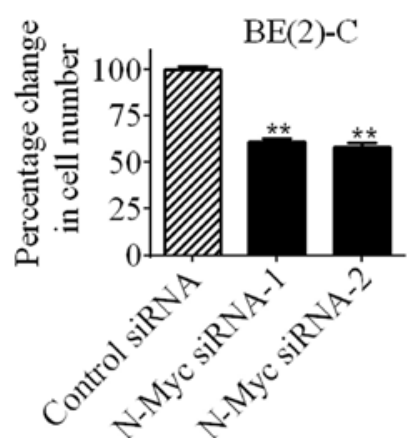

D

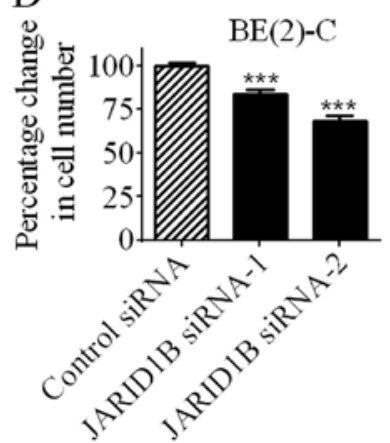

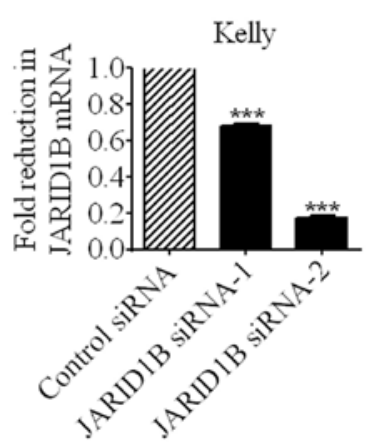

Kelly

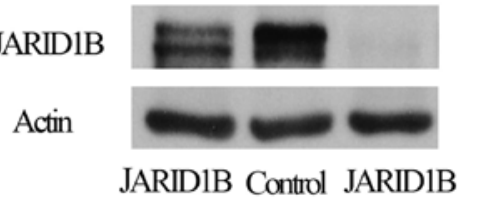

SiRNA-1 SiRNA SiRNA-2
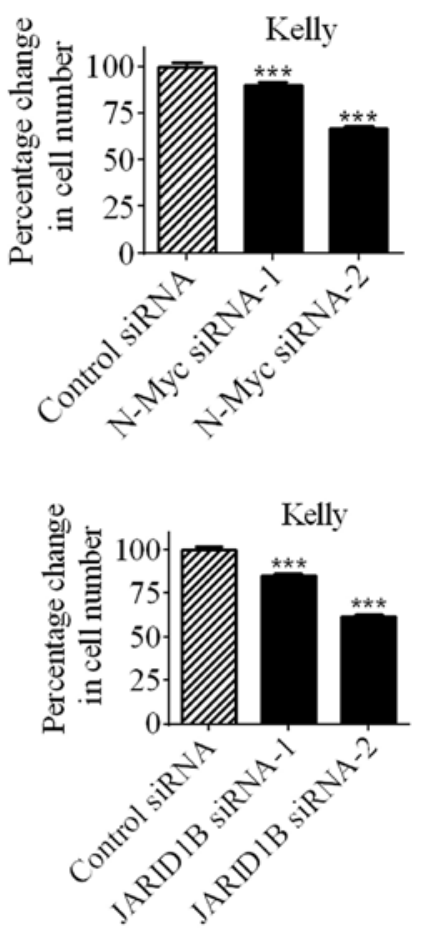

Figure 3. Repression of JARID1B expression reduces neuroblastoma cell proliferation. (A and B) BE(2)-C and Kelly cells were transfected with AllStars negative control siRNA, JARID1B siRNA-1 or JARID1B siRNA-2 for $48 \mathrm{~h}$, followed by RT-PCR and immunoblot analysis of (A) JARID1B mRNA and (B) protein expression. (C and D) $\mathrm{BE}(2)-\mathrm{C}$ and Kelly cells were transfected with AllStars negative control siRNA, N-Myc siRNA-1, N-Myc siRNA-2, JARID1B siRNA-1 or JARID1B siRNA-2 for $72 \mathrm{~h}$, followed by Alamar blue assays. The effects of (C) N-Myc siRNAs and (D) JARID1B siRNAs on cell proliferation were expressed as percentage changes in the numbers of cells after transfection with N-Myc siRNAs or JARID1B siRNAs, compared with control siRNA. Error bars represent standard error. ${ }^{* * *} \mathrm{P}<0.01 ;{ }^{* * * *} \mathrm{P}<0.001$.

JARID1B gene promoter region matching the Sp1-binding siteenriched region in K562 leukemia cells. We next performed ChIP assays with an anti-N-Myc antibody or a control IgG

and PCR with primers targeting -109 bp to -9 bp upstream of the JARID1B gene transcription start site or a negative control region. As shown in Fig. 2C, our own ChIP assays showed that the anti-N-Myc antibody efficiently immunoprecipitated the proximal JARID1B gene core promoter enriched of Sp1-binding sites in BE(2)-C neuroblastoma cells. Taken together, the data indicate that $\mathrm{N}-\mathrm{Myc}$ reduces JARID1B gene expression by direct binding to the Spl-binding site-enriched region of the JARID1B gene core promoter.

Repression of JARIDIB expression reduces neuroblastoma cell proliferation. To understand whether repression of JARID1B expression contributed to an N-Myc-regulated cancer phenotype, we first validated the efficacy of two independent siRNAs targeting different regions of JARID1B mRNA. RT-PCR and immunoblot analyses showed that both JARID1B siRNA-1 and JARID1B siRNA-2 knocked down JARID1B mRNA and protein expression (Fig. 3A and B). We then transfected BE(2)-C and Kelly cells with control siRNA, N-Myc siRNA-1, N-Myc siRNA-2, JARID1B siRNA-1 or JARID1B siRNA-2 for $72 \mathrm{~h}$, followed by Alamar Blue assays. As shown in Fig. 3C, knocking down N-Myc gene expression with N-Myc siRNA-1 or N-Myc siRNA-2 reduced the numbers of BE(2)-C and Kelly cells. Similarly, knocking down JARID1B gene expression with JARID1B siRNA-1 or JARID1B siRNA-2 also reduced the numbers of $\mathrm{BE}(2)-\mathrm{C}$ and Kelly cells (Fig. 3D). It is also noteworthy that JARID1B siRNAs did not induce neurite outgrowth, an indicator of neuroblastoma cell differentiation (negative data not shown). The data suggest that JARID1B induces neuroblastoma cell proliferation, although N-Myc oncoprotein represses JARID1B expression.

\section{Discussion}

Myc oncoproteins induce tumor initiation and promote tumor progression by transcriptional repression of tumor suppressor genes and transcriptional activation of oncogenes, and are overexpressed in $>50 \%$ of tumor tissues from the general population of cancer patients. We previously showed that Myc oncoproteins repress gene transcription by forming transcriptional repressor complexes with histone deacetylases at Sp1-binding sites of target gene promoters (5-8). By analyzing 35 histone marks after genomic binding by c-Myc, Guccione et al (10) revealed that histone H3K4 tri-methylation at Myc-responsive elements of target gene promoters is a strict prerequisite for Myc-induced transcriptional activation. However, the mechanism through which histone H3K4 is trimethylated during Myc-regulated transcriptional activation is unknown.

JARID1B specifically demethylates tri- and di-methylated forms of H3K4. In the present study, we examined published microarray gene expression data from other groups as well as our own, and found that JARID1B is one of the genes most significantly reactivated in a variety of cancer cells after transfection with siRNAs targeting the histone deacetylase SIRT1, SIRT2, HDAC2, N-Myc or treatment with the BET bromodomain inhibitor JQ1, which exerts anticancer effects by repressing N-Myc and c-Myc gene transcription $(17,19,20)$. Real-time RT-PCR and immunoblot studies confirmed that $\mathrm{N}-\mathrm{Myc}$ reduces JARID1B expression. Bioinformatics analyses 
show that the JARID1B gene core promoter is enriched of Sp1-binding sites, and a publically available ChIP-sequencing dataset shows that c-Myc binds to the Sp1-binding siteenriched region of the JARID1B gene core promoter in K562 leukemia cells. Consistently, our own ChIP assays showed that an anti-N-Myc antibody efficiently immunoprecipitates the JARID1B gene core promoter enriched in Sp1-binding sites in neuroblastoma cells. Taken together, the data suggest that N-Myc represses JARID1B gene transcription by direct binding to the Sp1-binding site-enriched region of the JARID1B gene promoter, most likely through recruiting histone deacetylases.

JARID1B has been shown to play a dual role in cancer. JARID1B expression is significantly downregulated in advanced and metastatic melanoma tissues, compared with normal melanocytic nevi (21). Using JARID1B as a biomarker, a small subpopulation of JARID1B low-expressing melanoma cells, which give rise to a highly proliferative progeny, has been characterized. Moreover, knocking down of JARID1B leads to an initial acceleration of melanoma growth (22). By contrast, JARID1B is overexpressed in bladder, lung, breast and prostate tumors at both mRNA and protein levels $(12,23,24)$, and knocking down JARID1B expression induces growth inhibition in cell lines derived from these cancers $(12,23,24)$. Our results showed that knocking down JARID1B expression reduces neuroblastoma cell proliferation, further supporting the findings in bladder, lung, breast and prostate cancer. As $\mathrm{N}-\mathrm{Myc}$ induces neuroblastoma cell proliferation and represses JARID1B expression, our data suggest that N-Myc-mediated transcriptional repression of JARID1B counterintuitively reduces tumor cell proliferation.

In summary, the present study showed that JARID1B expression is commonly repressed by Myc oncoproteins and histone deacetylases in various cancer cell lines, that $\mathrm{N}-\mathrm{Myc}$ represses JARID1B expression by direct binding to the Sp1-binding site-enriched region of the JARID1B gene promoter and that transcriptional repression of JARID1B counterintuitively reduces neuroblastoma cell proliferation. While reducing or eliminating JARID1B protein has been proposed to reduce c-Myc-induced tumors $(16,25)$, our study suggests that N-Myc-mediated transcriptional repression of JARID1B counterintuitively inhibits N-Myc-regulated cell proliferation and potentially tumorigenesis.

\section{Acknowledgements}

This study was supported by the National Health and Medical Research Council and Cancer Council New South Wales project grants. T.L. is a recipient of an ARC Future Fellowship. Children's Cancer Institute Australia is affiliated with the University of New South Wales and Sydney Children's Hospital.

\section{References}

1. Brodeur GM: Neuroblastoma: biological insights into a clinical enigma. Nat Rev Cancer 3: 203-216, 2003.

2. Maris JM and Matthay KK: Molecular biology of neuroblastoma. J Clin Oncol 17: 2264-2279, 1999.
3. Patel JH, Loboda AP, Showe MK, Showe LC and McMahon SB: Analysis of genomic targets reveals complex functions of MYC. Nat Rev Cancer 4: 562-568, 2004.

4. Eilers M and Eisenman RN: Myc's broad reach. Genes Dev 22: 2755-2766, 2008.

5. Liu T, Tee AE, Porro A, et al: Activation of tissue transglutaminase transcription by histone deacetylase inhibition as a therapeutic approach for Myc oncogenesis. Proc Natl Acad Sci USA 104: 18682-18687, 2007

6. Marshall GM, Gherardi S, Xu N, et al: Transcriptional upregulation of histone deacetylase 2 promotes Myc-induced oncogenic effects. Oncogene 29: 5957-5968, 2010.

7. Marshall GM, Liu PY, Gherardi S, et al: SIRT1 promotes N-Myc oncogenesis through a positive feedback loop involving the effects of MKP3 and ERK on N-Myc protein stability. PLoS Genet 7: e1002135, 2011.

8. Sun Y, Liu PY, Scarlett CJ, et al: Histone deacetylase 5 blocks neuroblastoma cell differentiation by interacting with N-Myc. Oncogene: Jul 1, 2013 (Epub ahead of print).

9. Liu PY, Xu N, Malyukova A, et al: The histone deacetylase SIRT2 stabilizes Myc oncoproteins. Cell Death Differ 20: 503-514, 2013.

10. Guccione E, Martinato F, Finocchiaro G, et al: Myc-binding-site recognition in the human genome is determined by chromatin context. Nat Cell Biol 8: 764-770, 2006.

11. Seward DJ, Cubberley G, Kim S, et al: Demethylation of trimethylated histone H3 Lys4 in vivo by JARID1 JmjC proteins. Nat Struct Mol Biol 14: 240-242, 2007.

12. Xiang Y, Zhu Z, Han G, et al: JARID1B is a histone H3 lysine 4 demethylase up-regulated in prostate cancer. Proc Natl Acad Sci USA 104: 19226-19231, 2007.

13. Schmitz SU, Albert M, Malatesta M, et al: Jarid1b targets genes regulating development and is involved in neural differentiation. EMBO J 30: 4586-4600, 2011.

14. Cellot S, Hope KJ, Chagraoui J, et al: RNAi screen identifies Jaridlb as a major regulator of mouse HSC activity. Blood 122: 1545-1555, 2013

15. Liu T, Liu PY, Tee AEL, et al: Over-expression of clusterin is a resistance factor to the anti-cancer effect of histone deacetylase inhibitors. Eur J Cancer 45: 1846-1854, 2009.

16. Secombe J, Li L, Carlos L and Eisenman RN: The Trithorax group protein Lid is a trimethyl histone $\mathrm{H} 3 \mathrm{~K} 4$ demethylase required for dMyc-induced cell growth. Genes Dev 21: 537-551, 2007.

17. Delmore JE, Issa GC, Lemieux ME, et al: BET bromodomain inhibition as a therapeutic strategy to target c-Myc. Cell 146: 904-917, 2011.

18. Banerjee C, Archin N, Michaels D, et al: BET bromodomain inhibition as a novel strategy for reactivation of HIV-1. J Leukoc Biol 92: 1147-1154, 2012.

19. Ott CJ, Kopp N, Bird L, et al: BET bromodomain inhibition targets both c-Myc and IL7R in high-risk acute lymphoblastic leukemia. Blood 120: 2843-2852, 2012.

20. Zuber J, Shi J, Wang E, et al: RNAi screen identifies Brd4 as a therapeutic target in acute myeloid leukaemia. Nature 478: 524-528, 2011.

21. Roesch A, Becker B, Meyer S, et al: Retinoblastoma-binding protein 2-homolog 1: a retinoblastoma-binding protein downregulated in malignant melanomas. Mod Pathol 18: 1249-1257, 2005.

22. Roesch A, Fukunaga-Kalabis M, Schmidt EC, et al: A temporarily distinct subpopulation of slow-cycling melanoma cells is required for continuous tumor growth. Cell 141: 583-594, 2010.

23. Hayami S, Yoshimatsu M, Veerakumarasivam A, et al: Overexpression of the JmjC histone demethylase KDM5B in human carcinogenesis: involvement in the proliferation of cancer cells through the E2F/RB pathway. Mol Cancer 9: 59, 2010.

24. Barrett A, Madsen B, Copier J, et al: PLU-1 nuclear protein, which is upregulated in breast cancer, shows restricted expression in normal human adult tissues: a new cancer/testis antigen? Int J Cancer 101: 581-588, 2002.

25. Secombe J and Eisenman RN: The function and regulation of the JARID1 family of histone H3 lysine 4 demethylases: the Myc connection. Cell Cycle 6: 1324-1328, 2007. 